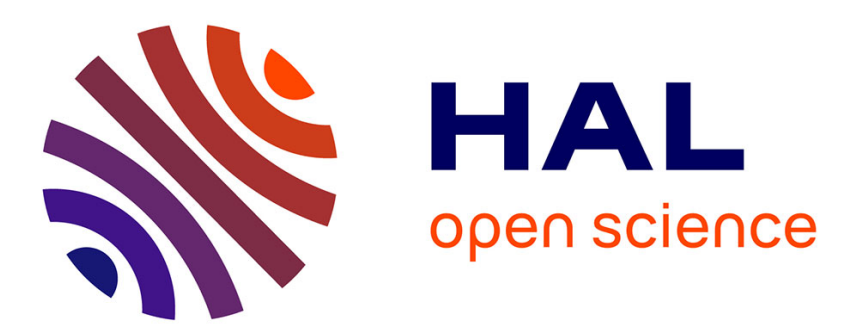

\title{
65nm CMOS Circuit Design of a Sampled Analog Signal Processor dedicated to RF Applications
}

\author{
Francois Rivet, Yann Deval, Jean-Baptiste Begueret, Dominique Dallet, \\ Philippe Cathelin, Didier Belot
}

\section{- To cite this version:}

Francois Rivet, Yann Deval, Jean-Baptiste Begueret, Dominique Dallet, Philippe Cathelin, et al.. 65nm CMOS Circuit Design of a Sampled Analog Signal Processor dedicated to RF Applications. Northeast Workshop on Circuits and Systems, Jun 2008, Montreal, Canada. pp.123-127. hal00269138

\section{HAL Id: hal-00269138 \\ https://hal.science/hal-00269138}

Submitted on 2 Apr 2008

HAL is a multi-disciplinary open access archive for the deposit and dissemination of scientific research documents, whether they are published or not. The documents may come from teaching and research institutions in France or abroad, or from public or private research centers.
L'archive ouverte pluridisciplinaire HAL, est destinée au dépôt et à la diffusion de documents scientifiques de niveau recherche, publiés ou non, émanant des établissements d'enseignement et de recherche français ou étrangers, des laboratoires publics ou privés. 


\title{
65nm CMOS Circuit Design of a Sampled Analog Signal Processor dedicated to RF Applications
}

\author{
Francois Rivet*, Yann Deval ${ }^{*}$, Jean-Baptiste Begueret*, Dominique Dallet*, Philippe Cathelin ${ }^{\dagger}$ and Didier Belot ${ }^{\dagger}$ \\ *Universite de Bordeaux 1, IMS Laboratory, 351 Cours de la Liberation, 33405 Talence Cedex, France \\ Email: \{francois.rivet,yann.deval,jean-baptiste.begueret,dominique.dallet\}@ims-bordeaux.fr \\ ${ }^{\dagger}$ STMicroelectronics, Central R\&D, 38926 Crolles Cedex \\ Email: \{philippe.cathelin,didier.belot\}@st.com
}

\begin{abstract}
The Software-Defined Radio (SDR) concept aims at designing a re-configurable radio architecture accepting all cellular or non-cellular standards working in the 0-5 $\mathbf{G H z}$ frequency range. Some technical challenges have to be solved in order to address this concept. A fully digital SDR system implying an A/D conversion close to the antenna is not feasible in the case of mobile terminal. This paper presents the design of an Analog Processor which process RF signal in order to select and convert into digital only the desired RF signal envelope. It uses the principle of a Fast Fourier Transform (FFT) to carry out basic analog functions with high accuracy at a low power consumption. Schematic and Post Layout Simulations are exhibited. Estimated die area and power consumption are numbered.
\end{abstract}

Index Terms - analog signal processing, software-defined radio, cognitive radio, sampled analog signal processor.

\section{INTRODUCTION}

$\mathbf{T}$ HE telecommunication industry claims for multifunctionnal mobile terminals. All kind of applications such as audio, graphic or video data are told to be integrated in a same wireless device. But they have to accommodate different wireless standards with different carrier frequencies, channel bandwidths, modulation schemes or data rates [1], [2].

The concept of Software-Defined Radio (SDR) can solve the problem of the wireless convergence. Reconfigurable circuits and systems are part of the solution as they can be tuned to any frequency band, select any reasonable channel bandwidth, and detect any known modulation. This paper exhibits the architecture and the schematic of a SDR RF Front-End dedicated to mobile terminals.

An ideal receiving SDR architecture is only sofware. It is composed of a Low Noise Amplifier (LNA), an Analog to Digital Converter (ADC) and a Digital Signal Processor (DSP). But, the resolution required for the Analog to Digital conversion is not feasible at a $10 \mathrm{GHz}$ frequency and a low power consumption required in the case of a mobile terminal [3], [4]. A mixed analog architecture was thus proposed to overcome the A/D-conversion bottleneck. A component called Sampled Analog Signal Processor (SASP) located between the LNA and the ADC processes the RF input signal (Fig. 2). It carries out operations on discrete-time analog voltage samples. The signal is processed analogically and its data rate is decreased to be converted into digital at a lower frequency, claiming a less-powerful ADC. Whereas the digital conversion technological bottleneck is avoided, new challenges appear in the analog domain.

The SASP is an analog processor performing an analog Discrete Fourier Transform (DFT) . It is ruled by :

- Two parameters inherited from the DFT equation (Eq. 1) to master the SDR requirements such as reprogrammability and flexibility: the sampling frequency $f_{\text {sampling }}$ and the number of voltage samples $N$ are taken into account to determine the spectral accuracy $\left(\frac{f_{\text {sampling }}}{N}\right)$.

- Two basic analog operations: one operation was to delay the voltage samples. This is matematically translated by $z^{-1}$ in the $z$-domain. The second was to weight and calculate the samples. The combination of both operations gives the DFT calculation.

$$
\begin{aligned}
X(k) & =\frac{1}{N} \sum_{n=0}^{N-1} x(n) W_{N}^{n k}, \quad k=0,1, \ldots, N-1 \\
W_{N} & =e^{-j(2 \pi / N)}
\end{aligned}
$$

where $N$ is the number of samples and $x(n)$ is the input voltage sample value.

Once the spectrum processed and displayed by analog voltage samples, only the samples representing the desired RF signal envelope are converted into digital. The digital process is just a matter of recovering the modulated signal into baseband. This is compared to a frequency translation as the RF signal envelope is translated into baseband.

\section{The Sampled Analog Signal Processor}

The SASP is composed by 3 parts to calculate the FFT:

- The continuous-time signal pre-conditioning [5],

- The sampler and the FFT,

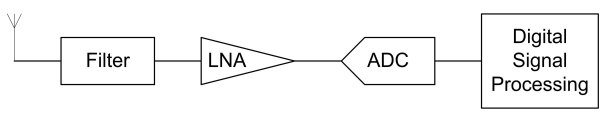

Fig. 1. Ideal SDR architecture

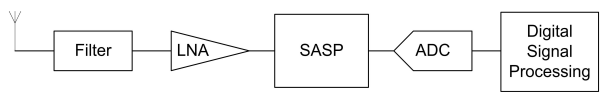

Fig. 2. Proposed SDR architecture 

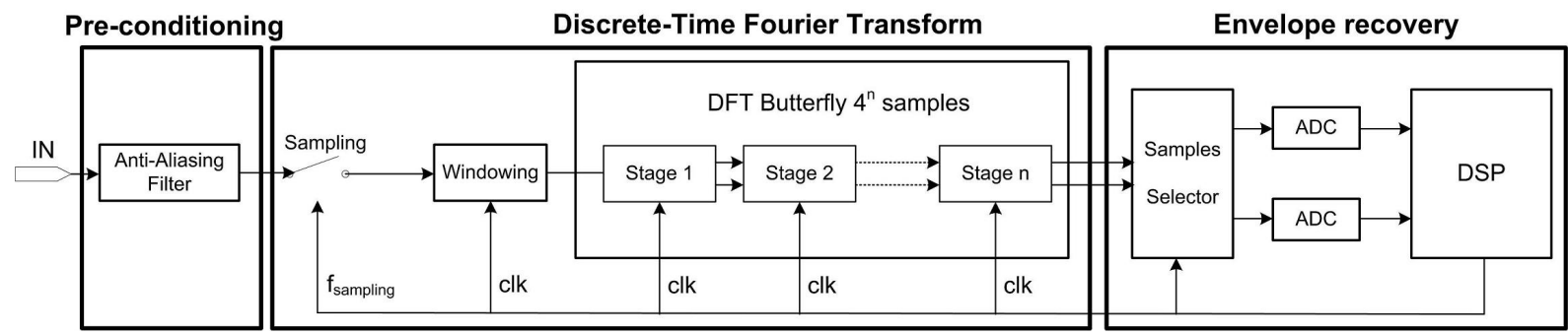

Fig. 3. SASP Architecture

- The envelope selection and conversion.

The SASP implements a pipeline FFT algorithm [6]. A radix-4 FFT using $\log _{4}(N)$ stages was chosen in order to improve the speed efficiency [7], [8]. All stages of a radix4 FFT use a basic module with 4 weighted inputs (twiddle factors) and 4 outputs.

A pipeline implementation consists in using one basic module per stage which runs with two processing phases [7], [8]:

1) Summation/substraction and weighting factor [10].

2) Feedback storage.

The basic stage architecture is composed by a delay line, two "switching boxes", and a processing unit [11] to process the basic analog operations on voltage samples. The only difference between each stage is the frequency clock of the "switching box" and the length of the delay line equal to $3.4^{n-k-1}$ samples, $\mathrm{k} \in\left[0 ; \log _{4}(N)-1\right]$ [8].

The technology used to design the circuit is $65 \mathrm{~nm}$ CMOS technology from STMicroelectronics. The analog implementation of the basic stage is composed by (Fig. 4):

- an input delay line (one for the real samples, and one for the imaginary samples),

- a weighting unit,

- a adding/substracting unit,

- a "feedback" delay line to serialize the samples at the stage output.

\section{A. The Delay Line}

The delay line processes a delay equivalent as a $z$ transformation to $z^{-1}$. It was carried out by an accumulation delay line that stores the voltage sample during a given time (Fig. 5). Three operating states of the delay line are performed. For example, at a given time:

1) One sample is loaded in a capacitor ( $S_{1}$ is closed).

2) One sample is stored during the delay time ( $S_{2}$ is open).

3) One sample is either carried out on the processing unit or on the output stage ( $S_{6}$ is closed).

A delay line is composed by $3.4^{n-k-1}$ delay cells, $\mathrm{k} \in$ $\left[0 ; \log _{4}(N)-1\right]$ (Fig. 7) [8]. A delay cell is composed by:

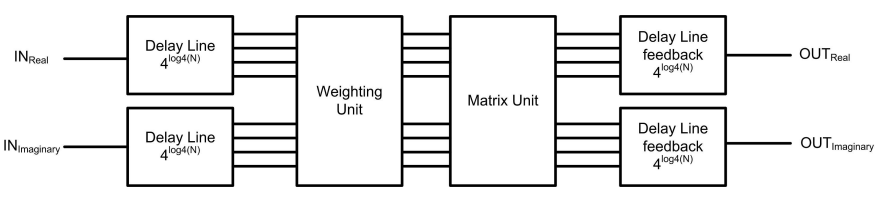

Fig. 4. Analog implementation of a Radix-4 FFT stage
- an input switch to load the voltage sample,

- a capacitor to store the voltage sample,

- an output switch to display the voltage sample.

Because the circuit runs mainly with charge transfers, DC offset errors are the main drawbacks induced (Eq. 2). Thus, a differential structure is chosen with both positive and negative signals centered on a DC voltage of $800 \mathrm{mV}$ with a linearity range of $200 \mathrm{mV}$. Only the transistors non linearities and the parasitic capacitors matters remain. These specifications rule the all specifications of the circuit.

$$
\text { Vout }=\operatorname{Vin} \underbrace{\left(1+\frac{\text { W.L.C. } C_{o x}}{C_{\text {hold }}}\right)}_{\text {non unity gain }}-\underbrace{\frac{W \cdot L \cdot C_{o x}}{C_{\text {hold }}}\left(V_{D D}-V_{T H}\right)}_{\text {pedestral error }}
$$

Each delay cell has a positive and a negative part to store the samples coming from the positive and the negative signals. An inside buffer protects the voltage samples against the output voltage variations and improves the charge transfer. It is implemented with a single transistor $M_{1,2}$ and a resistor $R_{1,2}$ (Fig. 6). It has a gain slightly superior to 1 to guarantee a gain of 2 along all the charge transfers to compensate the division by 2 provided by the FFT calculation. It has a linearity range of $200 \mathrm{mV}$ centered on the DC voltage of $800 \mathrm{mV}$.

A trade off is done on the capacitor value. It has to enable a fast load of the voltage sample without loosing charge during the delay time. As the basic structure of a delay cell is based on capacitor, the die area occupied by the capacitor in the

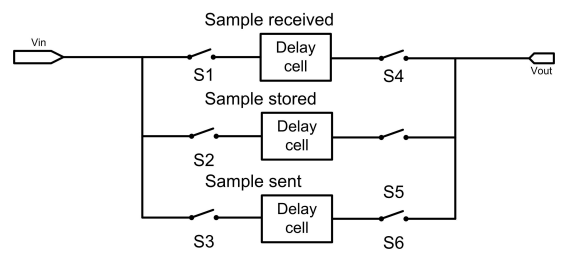

Fig. 5. Accumulation Delay Line

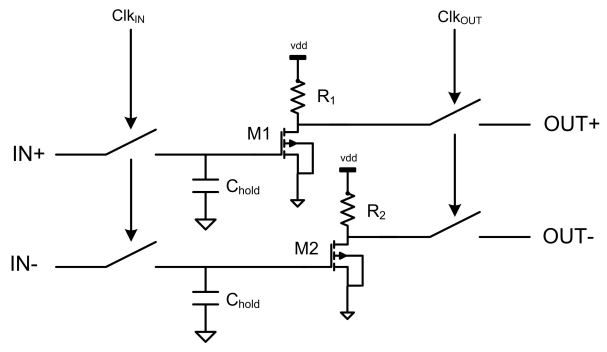

Fig. 6. Simplified schematic of a delay cell 
circuit is also a factor to take into account. The all SASP counts $4^{\log _{4} N}+4^{\log _{4} N-1}-2$ capacitors [8]. Thus, the trade off implies to choose a capacitor value as low as possible. The storage capacitor $C_{\text {hold }}$ was $50 \mathrm{fF}$ (Fig. 6).

Fig. 8 presented the simulation of a charge transfer. The acquisation time is $491 \mathrm{ps}$ at a sampling frequency of $500 \mathrm{MHz}$. The pedestral error is $0.11 \mathrm{mV}$ for a $30 \mathrm{mV}$ voltage sample. The inside buffer has a gain of 1.14 which leads to a total amplification of 2 during the charge transfer.

\section{B. The Matrix Unit (MU)}

MU adds and substracts voltage samples 4 by 4 as described in the algorithm matrix in [9]. It is composed by basic adders. Each adder is designed with 4 transistors connected to a common resistor (Fig. 9). Each transistor has a size of $\frac{W}{L}=$ 20. The current crossing each transistor is proportionnal to the input voltage equal to $V_{g s}$. The current crossing the resistor is the sum of the 4 currents coming from each transistors. Thus, the voltage seen at the drain of the transistors is proportionnal to the sum of the input voltages. As the circuit is differential, the structure substracts voltage samples by inverting some of the positive signal input with the negative ones. In Fig. 9, IN3 et IN4 are inverted. Voltage samples on IN3 and IN4 are thus substracted to those on IN1 and IN2. The Matrix contains adders et mixed adders/substracters to carry out the FFT calculation.

\section{The Weighting Unit (WU)}

WU weights each sample with a coefficient $W_{n}^{k}=\cos (2 \pi . n k)+i \cdot \sin (2 \pi . n k)$ (Eq. 1). The real part of the signal is weighted by $\cos (2 \pi . n k)$ and the imaginary by $\sin (2 \pi . n k)$ (Fig. 4). Every discrete sample is consequently weighted by a factor within the interval $[0,1]$. The principle of the voltage/current/voltage conversion

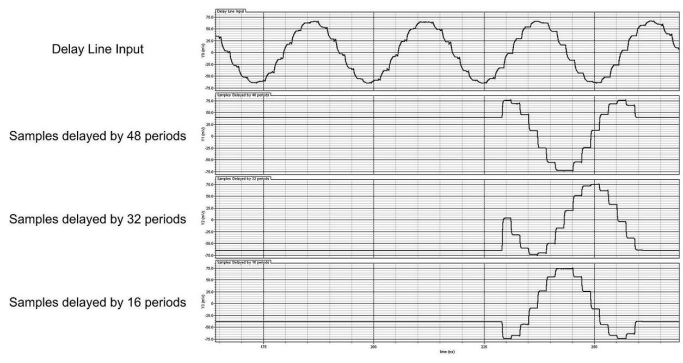

Fig. 7. Simulation of a delay line

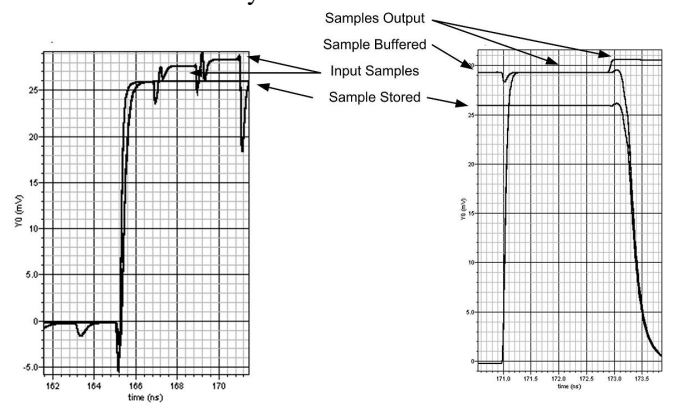

Fig. 8. Charge Transfer. Load and Display of a voltage sample
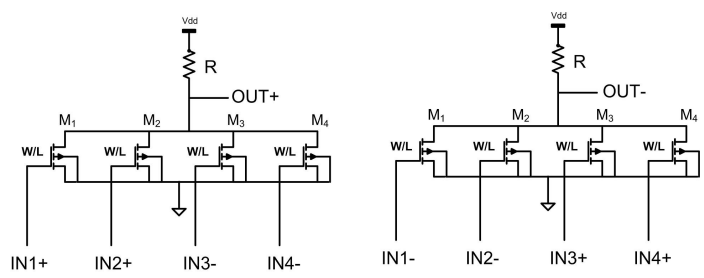

Fig. 9. Simplified schematic of a 4-voltage sample adder

coming from the matrix is used to carry out this analog operation. A switch network $\left(S_{x}\right)$ selects the input voltage of each transistor gate (Fig. 10). The input voltage can be either a voltage sample to be weighted or the DC reference voltage $(800 \mathrm{mV})$. Tab. I depicts the possible configuration of teh switch network. Every transistor $\left(M_{x}\right)$ has a different size. It implies that the current crossing each transistor is no more proportionnal to the input voltage but to the width of the transistor.

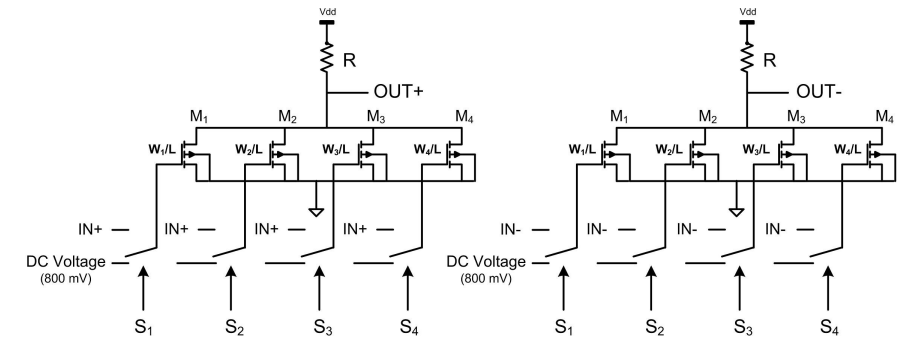

Fig. 10. Simplified schematic of the Weighting Unit

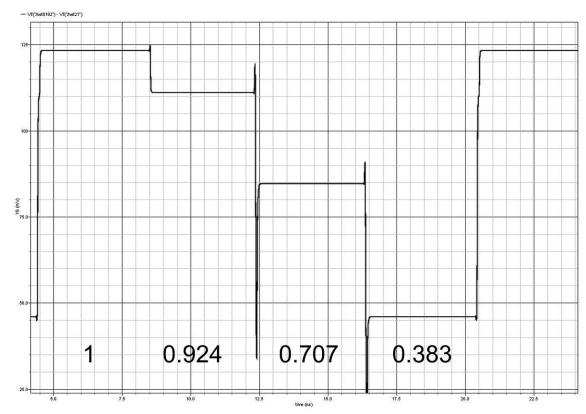

Fig. 11. A $100 \mathrm{mV}$ voltage sample weighted by 4 factors

Fig. 11 exhibits the weighting a $100 \mathrm{mV}$ voltage sample as shown in Tab. I. For instance, if $M_{3}$ and $M_{4}$ are switched on the input signal and $M_{1}$ and $M_{2}$ on the $800 \mathrm{mV}$ DC voltage reference, the voltage sample is weighted by 0.707 .

\section{The Feedback Delay Line}

The Feedback delay line had the same structure of the input delay line. It receives 4 by 4 the voltage samples throught 4 inputs and serializes all of them on a single output.

\section{RESULTS}

The circuit considered here is a 64-point radix-4 FFT processor. There are $\log _{4}(64)=3$ stages. It carried out an Analog FFT working at $500 \mathrm{MHz}$ to target the processing of RF signals in the $0-250 \mathrm{MHz}$ frequency range. A Post Layout Simulation 
TABLE I

Weighting Unit CoEFFicients APPLiCATION

\begin{tabular}{|c|c|c|c|c|c|c|c|}
\hline \multicolumn{4}{|c|}{ Switch Network Configuration } & \multicolumn{4}{|c|}{ Coefficients } \\
\hline$S_{1}$ & $S_{2}$ & $S_{3}$ & $S_{4}$ & & & & \\
\hline \multicolumn{4}{|c|}{ Transistor Characteristics } & \multicolumn{4}{|c|}{ For a $100 \mathrm{mV}$ input voltage sample simulation } \\
\hline$M_{1} \frac{W_{1}}{L}=2.1$ & $M_{2} \frac{W_{2}}{L}=5.3$ & $M_{3} \frac{W_{3}}{L}=7.8$ & $M_{4} \frac{W_{4}}{L}=9.3$ & $\cos (2 \pi \cdot n k)$ & Output & Effective Coefficient & Error \\
\hline $\mathrm{DC}$ & $\mathrm{DC}$ & $\mathrm{DC}$ & $\mathrm{DC}$ & 0 & 0 & 0 & $0 \%$ \\
\hline DC & $\mathrm{DC}$ & $\mathrm{DC}$ & IN & 0.383 & $45.9 \mathrm{mV}$ & 0.372 & $2.87 \%$ \\
\hline$\overline{\mathrm{DC}}$ & $\overline{\mathrm{DC}}$ & IN & IN & 0.707 & $84.5 \mathrm{mV}$ & 0.686 & $2.97 \%$ \\
\hline $\mathrm{DC}$ & IN & IN & IN & 0.924 & $111 \mathrm{mV}$ & 0.901 & $2.48 \%$ \\
\hline IN & IN & IN & IN & 1 & $123.1 \mathrm{mV}$ & 1 & $0 \%$ \\
\hline
\end{tabular}

TABLE II

(PLS) exhibited the FFT delivered by the SASP (Fig. 12). The input signal is a sinusoid at $39 \mathrm{MHz}\left(\frac{5 * f_{\text {sampling }}}{64}\right)$. The spectrum processed can be identified as pulses of the Fourier Transform of a sinewave. The characteristics of the 64-point circuit is shown in Tab. reftab:charac with a $1.2 \mathrm{~V}$ supply voltage. A layout view of the first stage is presented in Fig 13. Three parts of the stage schematic can be recognized: the input delay line, the matrix unit, the feedback delay lines. The perpectives are to display a SASP dedicated to a RF Front-End processor. It intents to carry out at least a 4096point analog FFT, working at $5 \mathrm{GHz}$. Technological and design improvements will lead to a power consumption under $500 \mathrm{~mW}$ to design this circuit.

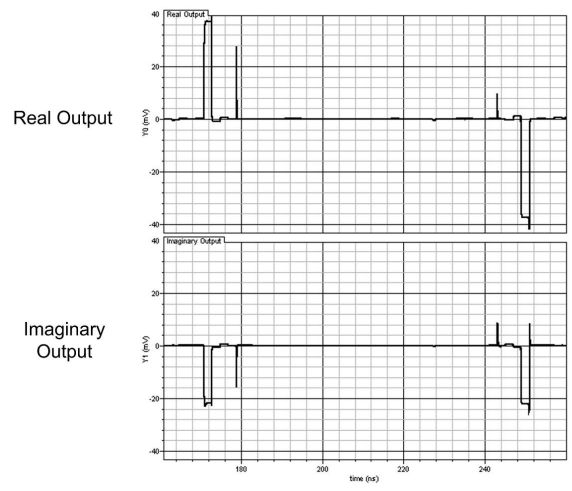

Fig. 12. Simulation of a 64-point SASP

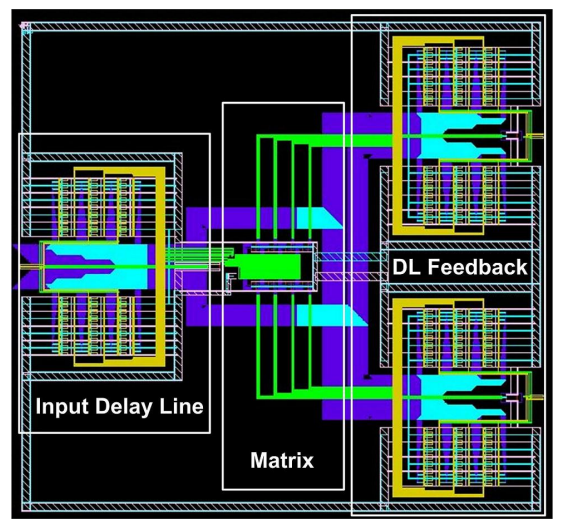

Fig. 13. Layout view of the first stage

\section{CONCLUSION}

The SASP is an Analog Processor dedicated to RF applications. It aims at lowering the RF signal frequencies by
64-POINT SASP CONSUMPTION AND DIE AREA

\begin{tabular}{|c|c|c|c|}
\hline \multirow{2}{*}{$\begin{array}{c}\text { Building } \\
\text { Block }\end{array}$} & \multicolumn{2}{|c|}{ Power Consumption } & Die Area \\
@ 500MHz & @ 1 GHz & $\left(\mathrm{mm}^{2}\right)$ \\
\hline \hline 4-sample delay line & $2,80 \mathrm{~mW}$ & $3,08 \mathrm{~mW}$ & $0.0126 \mathrm{~mm}^{2}$ \\
\hline 16-sample delay line & $11,22 \mathrm{~mW}$ & $12,35 \mathrm{~mW}$ & $0.0338 \mathrm{~mm}^{2}$ \\
\hline 64-sample delay line & $44,88 \mathrm{~mW}$ & $49,42 \mathrm{~mW}$ & $0.0481 \mathrm{~mm}^{2}$ \\
\hline 4-sample Weighting Unit & $9,24 \mathrm{~mW}$ & $9,24 \mathrm{~mW}$ & $0.0120 \mathrm{~mm}^{2}$ \\
\hline 16-sample Weighting Unit & $75,90 \mathrm{~mW}$ & $75,90 \mathrm{~mW}$ & $0.0523 \mathrm{~mm}^{2}$ \\
\hline Matrix Unit & $23,08 \mathrm{~mW}$ & $23,08 \mathrm{~mW}$ & $0.0060 \mathrm{~mm}^{2}$ \\
\hline \hline Stage 1 & $162,36 \mathrm{~mW}$ & $167,4 \mathrm{~mW}$ & $0.385 \mathrm{~mm}^{2}$ \\
\hline Stage 2 & $81,34 \mathrm{~mW}$ & $83,21 \mathrm{~mW}$ & $0.208 \mathrm{~mm}^{2}$ \\
\hline Stage 3 & $113,1 \mathrm{~mW}$ & $114 \mathrm{~mW}$ & $0.301 \mathrm{~mm}^{2}$ \\
\hline 64-point SASP & $372 \mathrm{~mW}$ & $378 \mathrm{~mW}$ & $3.1 \mathrm{~mm}^{2}$ \\
\hline
\end{tabular}

selecting the RF signal envelope. Only the desired signal envelope is converted into digital. Hence, the ADC worked at a dramatically reduced frequency and the A/D conversion technological bottleneck is avoided. This paper exhibited the circuit design of the SASP in a $65 \mathrm{~nm}$ CMOS technology. It draws the way to a Processor able to handle telecommunication signals, compatible with the SDR concept.

\section{REFERENCES}

[1] A. A. Abidi, "Evolution of a software-defined radio receivers rf frontend," in Proc. IEEE Radio Frequency Integrated Circuits Symposium (RFIC), San Francisco, USA, 2006, pp. 027-030.

[2] A. A. Abidi, "Software-defined radio receiver:dream to reality," IEEE Communications Magazine, pp. 111-118, 2006.

[3] G. Hueber, L. Maurer, G. Strasser, R. Stuhlberger, K. Chabrak, and R. Hagelauer, "Concept of a sdr compliant receive digital-front-end for cellular terminals," in Proc. IEEE IASTED International Conference, Krabi, Thailand, 2005, pp. 467-472.

[4] V. J. Arkesteijn, E. A. Klumperink, and B. Nauta, "An analogue frontend architecture for software defined radio," in Proc. IEEE ProRISC, Veldhoven, Netherlands, 2002.

[5] F. Rivet, Y. Deval, D. Dallet, JB. Begueret and D. Belot, "A Universal Radio Frequency Receiver Architecture Based on Sampled Analog Signal Processing," in Proc. IEEE NEWCAS'07, Montreal, Quebec, August 2007.

[6] F. Rivet, Y. Deval, D. Dallet, JB. Begueret and D. Belot, "A Disruptive Software-Defined Radio Receiver Architecture Based on Sampled Analog Signal Processing," in Proc. IEEE RFIC, Honolulu, USA, June 2007.

[7] E. Swartzlander, W. Young, and S. Joseph, "A radix 4 delay commutator for fast fourier transform processor implementation," IEEE Journal of Solid-State Circuits, vol. 19, pp. 702-709, Oct. 1984.

[8] A. El-Khashab, "Modular pipeline fast fourier transform algorithm," Ph.D. dissertation, University of Texas at Austin, 2003.

[9] F. Rivet, Y. Deval, D. Dallet, JB. Begueret, P. Cathelin and D. Belot, "A Disruptive Receiver Architecture Dedicated To Software Defined Radio," in IEEE TCAS-II, to be published, February 2008.

[10] E. Monastra and J. Huah, "Pipelined fast fourier transform processor," in US Patent number 5.038.311.

[11] S. Sayegh, "A pipeline processor for mixed-size FFT's," IEEE Trans. on Signal Processing, vol. 40, pp. 1892-1900, Aug. 1992. 